\title{
Bone morphogenetic protein 4 expression in multiple normal and tumor tissues reveals its importance beyond development
}

\author{
Emma-Leena Alarmo ${ }^{1,2}$, Heini Huhtala ${ }^{3}$, Tarja Korhonen ${ }^{4}$, Liisa Pylkkänen ${ }^{5,6}$, Kaija Holli ${ }^{5}$, \\ Tuula Kuukasjärvi ${ }^{7}$, Seppo Parkkila ${ }^{1,2,5}$ and Anne Kallioniemi ${ }^{1,2}$ \\ ${ }^{1}$ Institute of Biomedical Technology, University of Tampere and BioMediTech, Tampere, Finland; ${ }^{2}$ Fimlab \\ Laboratories, Tampere, Finland; ${ }^{3}$ School of Health Sciences, University of Tampere, Tampere, Finland; \\ ${ }^{4}$ Unit of Palliative Medicine, Tampere University Hospital, Tampere, Finland; ${ }^{5}$ School of Medicine, \\ University of Tampere, Tampere, Finland; ${ }^{6}$ Department of Oncology, University of Turku, Turku, Finland \\ and ${ }^{7}$ United Laboratories Ltd/Department of Pathology, Tampere University Hospital, Tampere, Finland
}

\begin{abstract}
Bone morphogenetic proteins (BMPs) are extracellular signaling molecules that belong to the transforming growth factor $\beta$ (TGF $\beta$ ) superfamily and are known to regulate cell proliferation, differentiation and motility, especially during development. BMP4 has an indispensable role in vertebrate development while limited information on BMP4 expression and function exists in adult tissues. Nevertheless, its contribution to cancer development and progression has gained increasing interest in recent years. Functional studies, especially in breast cancer, have implicated BMP4 both in inhibition of cell proliferation and in promotion of cell migration and invasion. To gain an insight into the function of BMP4 in normal and cancer tissues, BMP4 protein expression levels were analyzed by immunohistochemistry in 34 different normal organs/tissues, 34 different tumor types and finally in $\mathbf{4 8 6}$ breast cancer samples where possible associations between BMP4 and clinicopathological parameters were statistically evaluated. In over $20 \%$ of normal and malignant tissues, BMP4 was expressed at high level. Strong expression was observed particularly in some normal epithelial cells, such as bladder and stomach, and in squamous cell carcinomas. In breast cancer, strong BMP4 expression was detected in $25 \%$ of patients, and was associated with low proliferation index and increased frequency of tumor recurrence. Taken together, BMP4 is expressed in a subset of normal adult tissues and is likely to contribute to tissue homeostasis. However, in tumors, BMP4 expression levels vary considerably, implying diverse roles in different tumor types. This role is biphasic in breast cancer as BMP4 expression is linked to reduced proliferation and increased recurrence, thus corroborating our previous in-vitro functional data. Modern Pathology (2013) 26, 10-21; doi:10.1038/modpathol.2012.128; published online 17 August 2012
\end{abstract}

Keywords: bone morphogenetic protein 4; breast cancer; cancer; immunohistochemistry; normal tissue; protein expression; tissue microarray

Bone morphogenetic protein 4 (BMP4) is an extracellular signaling molecule that belongs to the family of bone morphogenetic proteins (BMPs) comprising the largest subfamily of the transforming growth factor $\beta$ (TGF $\beta$ ) superfamily. ${ }^{1}$ BMPs were originally named according to their bone inducing function, but they are also significant regulators of development. ${ }^{2,3}$ BMP4 is crucial for normal

Correspondence: Professor A Kallioniemi, MD, PhD, Institute of Biomedical Technology, University of Tampere, Biokatu 6, FM2, 33014 Tampere, Finland.

E-mail: anne.kallioniemi@uta.fi

Received 28 April 2012; revised 26 June 2012; accepted 26 June 2012; published online 17 August 2012 development as the homozygous deletion of Bmp4 in mice is embryonic lethal. ${ }^{4}$ The mouse embryos die between 6.5 and 9.5 days postcoitum and suffer from defects in gastrulation and mesoderm formation. ${ }^{3-5}$ Bmp4 expression is also detected later during organogenesis of the kidney, tooth, lung and gut as well as during skeletogenesis, limb patterning and mammary gland development. ${ }^{3,6,7}$

On a cellular level, BMPs are able to modulate cell proliferation, differentiation, survival, migration and even cell fate. ${ }^{3}$ This enormous pleiotropy stems from the intensive regulation of this signaling pathway. ${ }^{8,9}$ BMPs are synthesized as large precursor monomer proteins that are subsequently cleaved, dimerized and secreted as mature cytokine dimers 
outside the cell, or they may alternatively be stored in vesicles inside the cell. ${ }^{10}$ Mature BMP dimers bind to their specific transmembrane receptors to activate intracellular SMAD proteins that regulate target gene transcription. ${ }^{8}$ The BMP-SMAD pathway is also able to interconnect with other signaling pathways, and BMPs are known to activate the MAP kinase pathways independent of the SMAD proteins. ${ }^{9,10}$

The embryonic lethality caused by the loss of Bmp4 has impeded studies of BMP4 function in adult mammals. There have only been a few studies on the function of BMP4 in adult tissues and disease states other than cancer. BMP4 is involved in skeletal repair and the bone regeneration processes. ${ }^{11}$ In normal ovaries, BMP4 is expressed in a cell type-specific manner and is involved in dynamic changes during the ovarian cycle. ${ }^{12} \mathrm{Bmp} 4$ is expressed in the endocrine pancreas regulating insulin metabolism, and in the spleen during stressinduced erythropoiesis. ${ }^{13,14}$ Furthermore, BMP4 has been implicated in the idiopathic pulmonary fibrosis of the lung. ${ }^{15}$ Increased BMP4 expression has been observed in esophagitis and Barrett's esophagus where BMP4 induces the metaplastic transformation of squamous epithelial cells to columnar cells. ${ }^{16}$ These examples indicate that BMP4 clearly has a specific role in various processes in adult tissues and organs although comprehensive data on this issue are currently lacking.

BMP4 has been studied in several cancer types that originate from various tissues. ${ }^{17}$ In these studies, BMP4 has been functionally linked to the reduced growth of prostate, breast and pancreatic cancer cells, the increased migration and invasion of breast, colorectal, ovarian, melanoma and pancreatic cancer cells, the induction of the epithelialmesenchymal transition in ovarian and pancreatic cancer cells and to lung cancer cell senescence. ${ }^{18-25}$ Interestingly, $B M P 4$ has also recently been identified as a new susceptibility locus for colorectal cancer. ${ }^{26}$ BMP4 expression has been detected in various cancer cell lines and tumor cells, but often, the number of samples included in these studies has been low. BMP4 protein expression has also been detected in tumor samples from colorectal, gastric, hepatocellular, ovarian and renal carcinomas as well as in squamous cell carcinomas of the head and neck and melanomas. ${ }^{19,20,27-31}$ Yet, only a handful of studies have explored BMP4 expression within a particular tumor type. For example, in breast cancer, the information is truly limited. ${ }^{32}$ We have previously shown that $B M P 4$ is strongly expressed in breast cancer cell lines and primary breast tumors. ${ }^{23,33}$ Furthermore, we proposed a bidirectional function for BMP4 as in vitro it reduced cancer cell proliferation and yet was able to simultaneously increase the migration and invasion of breast cancer cells. ${ }^{23}$ Currently, no studies exploring BMP4 protein expression in breast tumors have been conducted.
In this work, we first systematically evaluated BMP4 protein expression in multiple normal and malignant tissues throughout the human body. Second, we studied BMP4 expression and its possible clinical significance in a large set of tumor tissues, including both ductal and lobular carcinomas, from breast cancer patients with extensive clinical data. BMP4 protein expression was visualized by immunohistochemistry in 34 different normal tissues, 34 different tumor types and 486 breast tumor samples.

\section{Materials and methods}

\section{Tissue Microarrays}

Three different tissue microarrays were used in this study. The normal human tissue array (MNO961) and the multiple organ tumor tissue array (MC2082) were obtained from US Biomax, Inc. (Rockville, MD). The tissue microarrays contained formalinfixed paraffin-embedded tissue samples with a core diameter of $1-1.5 \mathrm{~mm}$. The normal tissue array contained 96 tissue specimens from 34 different types of organs or anatomical sites either 1 sample (bone marrow and parathyroid), 2 samples (eye, pituitary gland, skin, spinal cord and spleen), 3 samples (adrenal gland, bladder, breast, cerebellum, cerebral cortex, colon, esophagus, fallopian tube, heart, liver, lung, ovary, pancreas, placenta, prostate, rectum, small intestine, stomach, striated muscle, testis, thymus, thyroid, tonsil, ureter, uterus cervix and uterus endometrium) or 6 samples (kidney) of each tissue type.

The tumor tissue array contained 208 tumor samples from 34 tumor types (including subtypes) and 20 different organs or anatomical sites. Tumors samples included eight cases each of adenocarcinoma of the stomach, colon, pancreas, endometrium and prostate, eight hepatocellular carcinomas, eight lymphomas of the spleen, eight seminomas, eight transitional cell carcinomas of the bladder, four esophagus adenocarcinomas and squamous cell carcinomas, four each of lung squamous cell carcinomas, adenocarcinomas, large cell and small cell carcinomas, four astrocytomas, four glioblastomas, four thyroid papillary and follicular carcinomas, four Hodgkin and non-Hodgkin lymphomas, eight ductal and lobular breast carcinomas, eight ovarian adenocarcinomas, four ovarian germ cell and stromal tumors, eight kidney clear cell and granular cell carcinomas, eight squamous cell carcinomas of the uterine cervix, eight squamous cell carcinomas of the head and neck, eight squamous cell carcinomas of the skin, four fibrosarcomas of the skin, four liposarcomas and eight malignant melanomas.

The breast cancer tissue microarray contained 243 invasive ductal carcinomas and 243 invasive lobular carcinomas that are part of a previously published 
larger study. ${ }^{33,34}$ Each tumor sample was represented by a single core biopsy with a diameter of $1 \mathrm{~mm}$ on the tissue microarray. The patient records contained information on clinicopathological parameters, primary treatments, recurrences and survival. The sites and times of recurrences as well as the patient survival information were updated on 30 October 2009. The mean age of the patients at the time of operation was 61 years (range 31-93). From the 486 patients, $83 \%$ were ER positive and $66 \%$ were PR positive (ER/PR status was missing in $2.5 \%$ of the cases). The grade distribution included $40 \%$ grade $1,46 \%$ grade 2 and $14 \%$ grade 3 with $0.2 \%$ of the cases not having available data. The pathological tumor stage was pT1 in 61\%, pT2 in 30\% and pT3-4 in $9 \%$ of the patients with data missing in $0.4 \%$ of the cases. The pathological lymph-node stage was negative ( $\mathrm{pN} 0$ ) in $56 \%$ of the patients and positive (pN1-2) in $38 \%$ of the patients with no available information for $6 \%$ of the cases. The pathological metastasis stage was positive (pM1) for 3\%, negative for $96 \%$ and missing for $1 \%$ of the patients. Altogether, $12 \%$ of the patients were ERBB2 positive (data missing in 5\% of the cases). The DNA content was diploid for $51 \%$ of the patients and either aneuploid or multiploid for $33 \%$ of the patients with $16 \%$ of the cases not having available data. The proliferation index information was obtained from patient records and had been determined either by flow cytometry or by Ki67 staining depending on the year of diagnosis. For flow-cytometric DNA content analysis, an S-phase fraction of $<7 \%$ was considered as low, $7-12 \%$ as intermediate and $>12 \%$ as high proliferation rate. For Ki67 staining, $<10 \%$ positive cells was considered as low, 10-15\% as moderate and $>15 \%$ as high proliferation. Altogether, the proliferation rate was low in $34 \%$, moderate in $23 \%$ and high in $19 \%$ of the patients with no information available for $24 \%$ of the cases. All except one of the patients had their primary tumors surgically removed and the majority of them with axillary clearance. Post-operative radiotherapy was given for $28 \%$ of the patients, $7 \%$ received adjuvant systemic treatment (endocrine therapy and/or chemotherapy), $37 \%$ of the patients were treated with both radiotherapy and systemic treatment, $28 \%$ received neither radiotherapy nor systemic treatment and in eight cases no treatment information was available. During the follow-up period, a local cancer recurrence was detected in $11 \%$ of the patients and a distant metastasis was found in $37 \%$ of the patients. The distribution of the metastasis sites is presented in Supplementary Table 1. The maximum follow-up period was 19.8 years, which contained information on the site and time of tumor recurrence and overall survival time. The use of the tumor samples and patient records in this study was approved by the Ethics Committee of Pirkanmaa Hospital District, the Ministry of Social Affairs and Health, and the National Authority for Medicolegal Affairs.

\section{BMP4 Immunohistochemistry}

BMP4 protein expression in tissue samples was studied with immunohistochemistry. Immunostaining was performed using an automated Ventana Benchmark staining system according to manufacturer's instructions (Ventana Medical Systems, Tuscon, AZ). The tissue microarray slides were first deparaffinized and rehydrated, followed by antigen retrieval (heat treatment in $10 \mathrm{mM}$ Tris-HCl, $1 \mathrm{mM}$ EDTA). BMP4 protein was detected with a mouse anti-BMP4 monoclonal antibody (clone 3H2, 1:10 dilution; Chemicon Millipore, Temecula, CA) and an ultraView Universal DAB Detection Kit (Ventana Medical Systems). The slides were counterstained with hematoxylin-eosin. A control with no BMP4 antibody showed no staining. To further verify the specificity of the BMP4 antibody, immunocytochemical staining of breast cancer cell lines with high (HCC38) and low/no (HCC1954) BMP4 mRNA expression ${ }^{23}$ was performed and revealed strong and no expression, respectively.

The entire tissue core for each sample was evaluated by pathologists, and the BMP4 staining was classified as negative, weak diffuse, moderate diffuse or strong granular staining. For normal tissues, each sample was classified based on the staining pattern observed in the majority (at least 75\%) of the cells except that samples with at least $10 \%$ of the cell showing granular staining were classified as strong granular. Data from different tissue compartments, eg, epithelium and stroma, were recorded separately when applicable. For cancer samples, the same scoring criteria were applied but only tumor cells were evaluated. The possible presence of leukocytes with high BMP expression was recorded separately and did not influence the scoring of the corresponding normal tissue or tumor samples.

\section{Statistical Analysis}

The significance of BMP4 expression in primary breast tumors was evaluated using statistical analyses (SPSS program, version 11.01; SPSS Inc., Chicago, IL). The associations of BMP4 expression with standard clinicopathological parameters and tumor recurrences were analyzed using Fisher's exact test. The Kaplan-Meier method and the logrank test were applied to estimate the differences between the BMP4 expression groups in the rate of first local and distant recurrences as well as the overall survival time, breast cancer-specific survival time and disease-free time.

\section{Results}

The Classification of BMP4 Expression in Tissue Samples

BMP4 protein expression was determined in formalinfixed paraffin-embedded normal and tumor tissue 

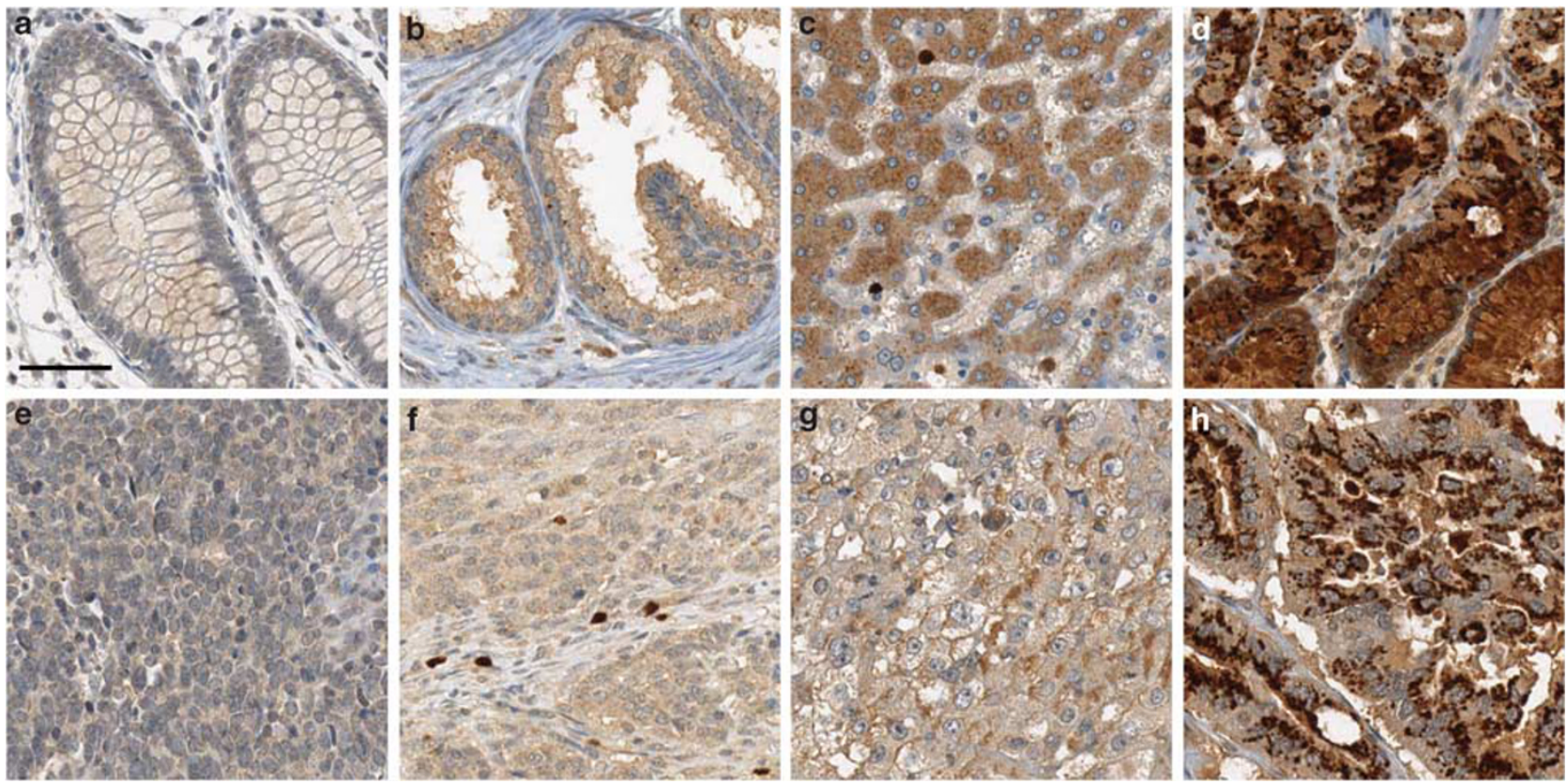

Figure 1 BMP4 expression in (a-d) normal tissue samples and (e-h) tumor samples. Representative examples of negative BMP4 expression in (a) normal colon and (e) small cell carcinoma of the lung, low diffuse staining of BMP4 in (b) normal prostate and (f) stomach adenocarcinoma, moderate diffuse staining in (c) normal liver and (g) endometrioid adenocarcinoma of the uterus, and strong granular staining in (d) normal stomach and (h) lung adenocarcinoma are shown. Liver tissue macrophages (Kupffer cells) are strongly stained in the liver, and leukocytes are strongly stained in the tumor sample of the stomach. The scale bar in (a) indicates $50 \mu \mathrm{m}$ and is the same for all images.

samples by immunohistochemistry. BMP4 expression was classified into four different staining categories: negative, weak diffuse, moderate diffuse and strong granular staining (Figure 1). In the diffuse staining category, BMP4 expression was detected evenly throughout the cytoplasm, whereas in the strong granular staining category, distinct granules were evident in the cytoplasm possibly suggesting a secretory expression pattern. Thus, the BMP4 staining could also be categorized into nongranular and granular patterns.

\section{BMP4 Expression in Normal Tissues}

BMP4 protein expression was first explored in the normal tissues. Altogether, of the 34 tissues or organ sites evaluated, 9 (26\%) were considered as negative for BMP4 expression and 5 (15\%) expressed BMP4 diffusely at a low level (Table 1; Figure 1a and b). Moderate diffuse staining was detected in two organs, the liver and parathyroid gland (Figure 1c).

Strong granular BMP4 expression was detected in 8 out of the 34 tissues (24\%) (Table 1; Figure 1d). In seven organs, the granular expression was detected particularly in the epithelial cells of the tissue. BMP4-positive granules were identified in the transitional epithelium of the bladder and ureter. Likewise, the stratified squamous epithelium was positive for BMP4 granules in the oral cavity and ectocervix. In the alimentary canal, the stratified squamous epithelium of the esophagus also strongly expressed BMP4. In the stomach, especially strong staining was detected in the epithelial cells of the surface mucosa and the chief cells of the base segment of the gastric glands, whereas the parietal cells clearly showed weaker staining. BMP4positive granules were also observed in the lung cells lining the alveoli. In non-epithelial tissues, granular BMP4 expression was identified in the red pulp area of the spleen.

Altogether, $15 \%(5 / 34)$ of the tissues could not be clearly classified into the four main staining categories (Table 1). A more specific staining pattern for BMP4, denoted as punctuated staining, was detected in the skeletal and cardiac muscles. In the cerebellum, cerebral cortex and spinal cord, BMP4 expression was detected only in cells that most likely represent the microglia of the central nervous system.

Five tissues and/or organ sites (15\%) showed variable staining between the samples. For example, granular BMP4 staining was identified in some, but not all samples, from tissues of the fallopian tube, kidney, endometrium and skin. In the kidney, strong BMP4 expression was detected in the collecting ducts, diffuse staining was detected in the proximal tubules and negative staining was observed in the glomeruli. Additionally, in the skin, granular BMP4 staining was observed particularly in the hair follicles.

Interestingly, throughout the tissue samples, strong BMP4 expression was observed in leukocytes (such as neutrophilic granulocytes in the placenta) and often in the tissue-specific macrophages (eg, the Kupffer cells of the liver) (Figure 1c). Overall, 21 out of the 34 tissue sites had positively stained 
Table 1 BMP4 expression in 34 organs or tissues

\begin{tabular}{|c|c|c|c|}
\hline \multirow[t]{2}{*}{ Organ/tissue } & $\begin{array}{c}\text { BMP4 } \\
\text { staining }\end{array}$ & \multirow[t]{2}{*}{$\begin{array}{l}\text { Special } \\
\text { pattern }\end{array}$} & $\begin{array}{l}P o s \\
L C^{\mathrm{a}}\end{array}$ \\
\hline & Neg Low Moderate Granular & & \\
\hline Adrenal gland & $\times$ & & $\times$ \\
\hline Bone marrow ${ }^{\mathrm{b}}$ & $\times$ & & \\
\hline GI-Small intestine & $\times$ & & $\times$ \\
\hline GI-Colon & $x$ & & $\times$ \\
\hline GI-Rectum & $x$ & & $x$ \\
\hline Ovary & $x$ & & \\
\hline Pancreas & $x$ & & $x$ \\
\hline Thymus & $x$ & & $x$ \\
\hline Thyroid & $\times$ & & $x$ \\
\hline
\end{tabular}

\begin{tabular}{lll}
\hline Eye $^{\mathrm{c}}$ & $\times$ & \\
Breast & $\times$ & $\times$ \\
Placenta & $\times$ & \\
Prostate & $\times$ & \\
Testis & $\times$ &
\end{tabular}

\begin{tabular}{lll}
\hline Liver & $\times$ & $\times$ \\
Parathyroid $^{b}$ & $\times$ & $\times$
\end{tabular}

\section{Bladder}

GI-Esophagus

GI-Stomach

Lung

Spleen $^{\mathrm{C}}$

Tonsil

Ureter

Uterus-cervix

\begin{tabular}{lcc}
\hline Heart $^{\mathrm{d}}$ & $\times$ & \\
Striated muscle $^{\mathrm{d}}$ & $\times$ & \\
Cerebellum $_{\text {Cerebral cortex }}$ & $\times$ & $\times$ \\
Spinal cord $^{\mathrm{C}}$ & $\times$ & $\times$ \\
\end{tabular}

\section{Pituitary gland ${ }^{\mathrm{c}}$}

Uterus-

endometrium

Fallopian tube

Kidney ${ }^{\mathrm{e}}$

Skin $^{\mathrm{C}}$

$\begin{array}{lll}\times & \times \\ \times & & \times \\ \times & \times & \times \\ \times & \times & \times \\ & \times & \times\end{array}$

BMP4 staining was classified as negative, low diffuse, moderate diffuse or strong granular. In the special pattern group, BMP4 staining was not classified into any of the four main groups.

${ }^{\mathrm{a} B M P} 4$ expression in leukocytes.

${ }^{\mathrm{b}}$ Number of samples, $n=1$.

${ }^{\mathrm{C}}$ Number of samples, $n=2$.

${ }^{\mathrm{d}}$ Punctuated staining pattern.

${ }^{\mathrm{e}}$ Number of samples, $n=6$.

leukocytes (Table 1). Yet, due to the intensive BMP4 staining, the exact identification of the leukocyte type was not possible. Interestingly, the lymphatic cell population in the tonsils was negative for BMP4 expression.

\section{BMP4 Expression in Tumor Tissues}

Next, we examined the BMP4 expression in the multiple organ tumor tissue microarray. Half of the tumor samples were either BMP4 negative $(14 \%)$ or

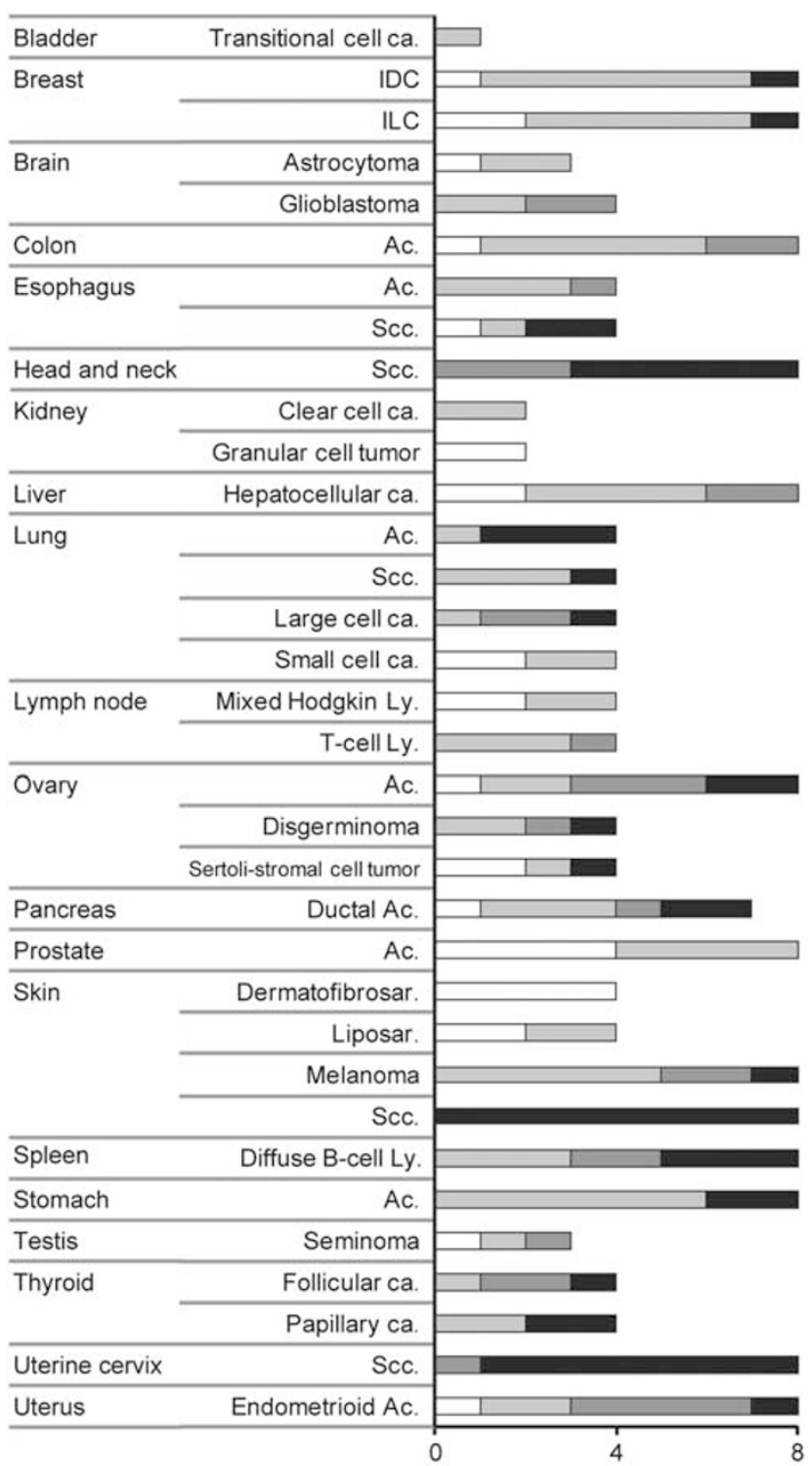

$\square 0 \square 1 \square 2 \square 3$

No. of samples

Figure 2 BMP4 expression in multiple tumor tissue arrays. Numerals indicate the strength of BMP 4 expression $(0=$ negative, $1=$ low, $2=$ moderate and $3=$ strong granular staining). ca., carcinoma; IDC, invasive ductal carcinoma; ILC, invasive lobular carcinoma; Ac., adenocarcinoma; Scc., squamous cell carcinoma; Ly., lymphoma; Sar., sarcoma.

expressed BMP4 at low level (37\%) (Figures 1e, f and 2). Moderate diffuse staining was detected in $14 \%$ of the samples and $22 \%$ had a strong granular BMP4 expression pattern (Figures 1g, $h$ and 2). In $13 \%$ of the samples, the BMP4 status could not be determined due to missing or unrepresentative samples.

The most notable common feature among the samples was the strong granular BMP4 expression in tumors arising from squamous cell epithelia particularly in the squamous cell carcinomas of the head and neck, skin and uterine cervix and to a lesser degree in the esophagus and lung (Figure 2). 

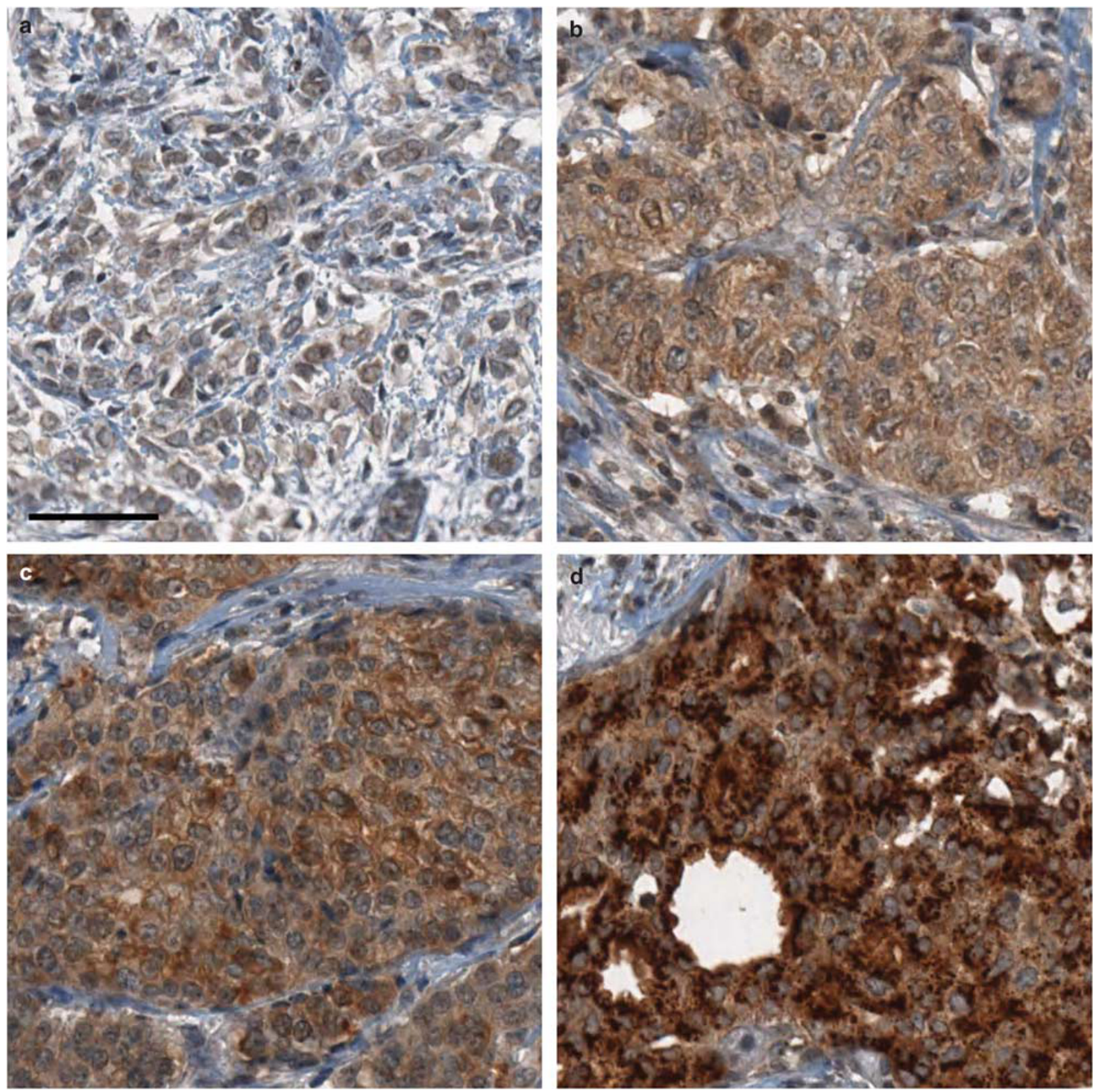

Figure 3 BMP4 expression in a breast cancer tissue microarray. Representative examples are shown for (a) negative, (b) weak diffuse, (c) moderate diffuse and (d) strong granular staining of BMP4. The scale bar in (a) indicates $50 \mu \mathrm{m}$ and is the same for all images.

In general, roughly half of the tumor types examined had BMP4 expression pattern that ranged from a negative/low to a strong level (Figure 2). In these tumor types, strong granular BMP4 expression was detected in less than half of the samples within a given cancer type (eg, ovary, pancreas and uterus). In approximately one-third of the tumor types, no BMP4 granular staining was observed (eg, brain, colon and prostate) (Figure 2).

Nine organs were represented by more than one histological tumor type in our survey. Specific differences in BMP4 expression within tumor subtypes were detected in the lung and skin. In the lung, $42 \%$ (5/12) of the non-small cell lung carcinoma samples (adenocarcinoma, squamous cell carcinoma and large cell carcinoma) had strong BMP4 granular expression, and none were negative. Contrarily, the small cell carcinoma samples were either BMP4 negative or displayed a low expression level (Figure 1e and h). Similarly, in the skin, the expression pattern also varied between tumor types as dermatofibrosarcomas and liposarcomas expressed BMP4 at lower level compared with the melanoma and squamous cell carcinoma samples (Figure 2). In the esophagus, granular expression was not detected in adenocarcinomas but only in 
squamous cell carcinomas. In contrast, no drastic differences were observed between tumor subtypes of the breast, ovary or thyroid. In these tumors, BMP4 was expressed in the majority of samples, and the expression patterns, ranging from weak to granular, were represented almost equally. Finally, mainly negative or weak BMP4 expression was detected in astrocytomas, glioblastomas and lymphomas originating from the lymph nodes.

The remaining tumors contained only one histological subtype on the tissue microarray. Nearly, all samples expressed BMP4 in four tumor types (ie, pancreas, spleen, stomach and uterus) even though strong granular BMP4 staining was observed in less than half of the samples. In three cancers, colon, liver and prostate, BMP4 expression was generally weak, and granular staining was not observed. The number of samples analyzed was very low for bladder, testicular and the two types of renal cancer.

Due to the relatively low number of samples for each tumor type, no conclusions were drawn between the expression of BMP4 and the available tumor characteristics. However, the observation that $\mathrm{BMP} 4$ expression in astrocytomas was lower than in glioblastomas was noteworthy as glioblastomas are considered to be a more severe form of brain tumor. Among the stromal cells, leukocytes in the tumor tissue were strongly positive for BMP4 similar to the normal tissue samples. Of note, there was no association between the presence of positively stained leukocytes and high BMP4 expression in the tumor tissue.

\section{BMP4 Expression in Breast Cancer}

Finally, a breast cancer tissue microarray that included 486 patient samples was utilized to study BMP4 expression. BMP4 expression was determined in $66 \%(314 / 486)$ of the tumors, of which 171 were invasive ductal carcinomas and 143 were invasive lobular carcinomas. The rest of the samples were either missing or unrepresentative. BMP4 expression was found to be negative or weak in $197(63 \%)$, moderately diffuse in $44(14 \%)$ or strongly granular in $73(23 \%)$ of the tumor samples (Figure 3). To study the association of BMP4 expression with standard clinicopathological parameters, the samples were divided into two groups: non-granular BMP4 expression $(n=241)$ and granular BMP4 expression $(n=73)$. Strong granular $\mathrm{BMP} 4$ staining was detected more often in the lobular carcinoma group compared with the ductal carcinoma group (64 vs 36\%, $P<0.001$ ) (Table 2). Patients with tumors containing BMP4 granular staining appeared to have more slowly proliferating cancers as a higher fraction of these tumors had low proliferation indexes compared with the nongranular tumors (52 vs $36 \%, P=0.019$ ) (Table 2). No further statistically significant associations were
Table 2 BMP4 expression and the standard clinicopathological parameters of 314 breast tumor samples.

\begin{tabular}{|c|c|c|c|c|c|}
\hline \multirow[t]{2}{*}{ Variable } & \multicolumn{2}{|c|}{$\begin{array}{l}\text { Non-granular } \\
\quad(\mathrm{n}=241)\end{array}$} & \multicolumn{2}{|c|}{$\begin{array}{c}\text { Granular } \\
(\mathrm{n}=73)\end{array}$} & \multirow[t]{2}{*}{$P$-value } \\
\hline & $\mathrm{n}$ & $\%$ & $\mathrm{n}$ & $\%$ & \\
\hline \multicolumn{6}{|l|}{ Tumor type } \\
\hline ILC & 96 & 40 & 47 & 64 & \multirow[t]{2}{*}{$<\mathbf{0 . 0 0 1}$} \\
\hline IDC & 145 & 60 & 26 & 36 & \\
\hline \multicolumn{6}{|l|}{ pT stage } \\
\hline pT1 & 146 & 61 & 48 & 66 & \multirow[t]{4}{*}{0.728} \\
\hline pT2 & 73 & 30 & 20 & 27 & \\
\hline pT3-pT4 & 21 & 9 & 5 & 7 & \\
\hline NA & 1 & & & & \\
\hline \multicolumn{6}{|l|}{$p N$ stage } \\
\hline pNo & 132 & 57 & 42 & 65 & \multirow[t]{4}{*}{0.578} \\
\hline pN1 & 89 & 39 & 21 & 32 & \\
\hline $\mathrm{pN} 2$ & 9 & 4 & 2 & 3 & \\
\hline NA & 11 & & 8 & & \\
\hline \multicolumn{6}{|l|}{ pM stage } \\
\hline pMo & 227 & 95 & 71 & 97 & \multirow{3}{*}{0.740} \\
\hline pM1 & 11 & 5 & 2 & 3 & \\
\hline NA & 3 & & & & \\
\hline \multicolumn{6}{|l|}{$E R^{\mathrm{a}}$} \\
\hline Positive & 199 & 85 & 60 & 82 & \multirow[t]{3}{*}{0.612} \\
\hline Negative & 36 & 15 & 13 & 18 & \\
\hline NA & 6 & & & & \\
\hline \multicolumn{6}{|l|}{$P R^{\mathrm{a}}$} \\
\hline Positive & 163 & 69 & 48 & 66 & \multirow[t]{3}{*}{0.562} \\
\hline Negative & 72 & 31 & 25 & 34 & \\
\hline NA & 6 & & & & \\
\hline \multicolumn{6}{|l|}{ Proliferation $^{\mathrm{b}}$} \\
\hline Low & 67 & 36 & 27 & 52 & \multirow[t]{4}{*}{0.019} \\
\hline Moderate & 65 & 35 & 8 & 15 & \\
\hline High & 53 & 29 & 17 & 33 & \\
\hline NA & 56 & & 21 & & \\
\hline \multicolumn{6}{|l|}{$E R B B 2^{\mathrm{a}}$} \\
\hline Positive & 30 & 13 & 5 & 7 & \multirow[t]{3}{*}{0.185} \\
\hline Negative & 202 & 87 & 65 & 93 & \\
\hline NA & 9 & & 3 & & \\
\hline \multicolumn{6}{|c|}{ Histological/nucleargrade } \\
\hline I & 81 & 33 & 27 & 38 & \multirow[t]{4}{*}{0.322} \\
\hline II & 115 & 48 & 37 & 51 & \\
\hline III & 45 & 19 & 8 & 11 & \\
\hline NA & & & 1 & & \\
\hline \multicolumn{6}{|l|}{$D N A$} \\
\hline Diploid & 109 & 52 & 35 & 61 & \multirow[t]{3}{*}{0.227} \\
\hline Aneu/Multiploid & 99 & 48 & 22 & 39 & \\
\hline NA & 33 & & 16 & & \\
\hline
\end{tabular}

NA, not available.

${ }^{a}$ ER, PR and ERBB2 statuses were determined by immunohistochemistry.

broliferation activity was determined by flow cytometry (S-phase fraction $<7 \%$ low, $7-12 \%$ moderate and $>12 \%$ high), or Ki67 staining (Ki67 $<10 \%$ low, $10-15 \%$ moderate and $>15 \%$ high).

$P$-values $<0.05$ are shown in bold.

found between BMP4 expression and other standard clinicopathological parameters (eg, ER/PR status, grade, TNM stage, DNA content or ERBB2 status). 

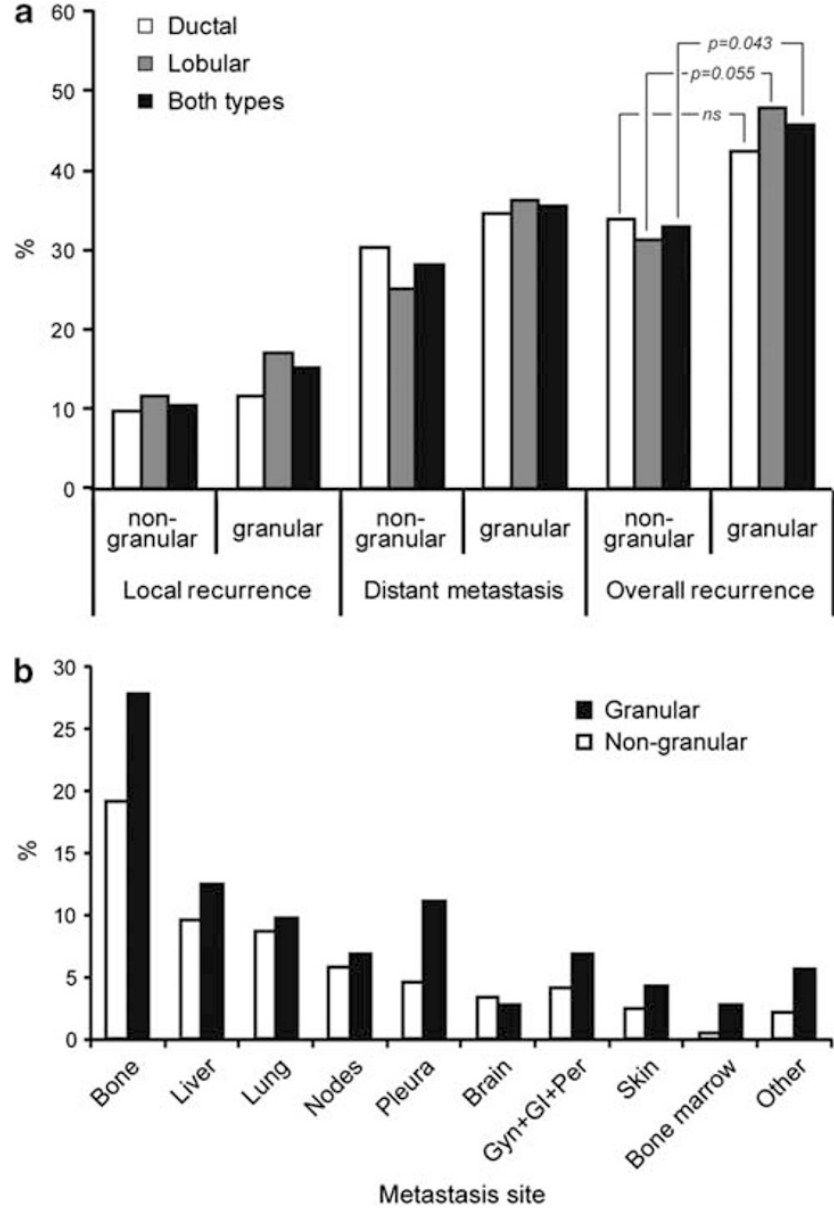

Figure 4 BMP4 expression correlates with breast tumor recurrence. (a) The percentages of patients that suffered from local, distant and overall recurrences are shown. A $P$-value of $<0.05$ was considered to be statistically significant (NS, not significant). (b) The percentages of patients with BMP4 granular or nongranular primary tumors that had distant metastases at a particular organ site are shown.

Next, the association between BMP4 expression and tumor recurrence was evaluated. Patients with tumors containing strong granular BMP4 staining suffered from overall tumor recurrence, including both local recurrences and distant metastases, more often than patients with tumors displaying non-granular BMP4 staining (49 vs 33\%, $P=0.043$ ) (Figure 4a). This association was subsequently explored in each tumor type separately. Among lobular carcinomas, the tumor recurrence was even more prominent within patients with granular tumors (48 vs $31 \%, P=0.056$ ) (Figure 4a). Yet, due to the low total number of patients with tumor recurrence, this difference was not statistically significant (Figure 4a). We then explored whether the observed increase in recurrence from tumors with BMP4 granular staining was reflected in particular sites of metastasis (eg, bone, liver, lung, nodes, pleura, brain, gynecological organs, GI tract, peritoneum, skin and bone marrow). In general, patients with tumors displaying BMP4 granular staining more often showed metastasis at all sites (Figure 4b). Metastases were most prevalently detected in bone tissue (BMP4 non-granular 19\% vs BMP4 granular 28\%) (Figure 4b). Nevertheless, no differences were statistically significant, which was likely again due to the low number of events evaluated.

Finally, the potential association between BMP4 expression and patient survival was explored. The overall survival and breast cancer-specific survival did not differ between patients with tumors displaying granular or non-granular BMP4 staining. Since granular BMP4 staining was associated with low proliferation rate, we divided the patients further into subgroups based on the proliferation status but again found no statistically significant associations. Time-to-event analyses were also performed for the time to first metastasis, local recurrence, first bone metastasis and disease-free time. No statistically significant differences were observed for any of the above analyses. In addition, the associations between BMP4 expression and the use of hormone-replacement therapy or BMP7 expression status were also examined, but no differences were found between tumors with granular or non-granular BMP4 staining.

\section{Discussion}

BMP4 is a well-known developmental regulator that is widely expressed during early embryogenesis. ${ }^{5}$ However, considerably less is known about BMP4 expression and function in normal adult tissues. Genes and signaling pathways that normally participate in developmental processes have been shown to also be reactivated in cancer pathogenesis. ${ }^{36}$ Thus, BMP4 and other BMP family members have begun to be a common research topic in the cancer field. Interestingly, BMP2 and BMP7 are currently used in the clinic for healing bone fractures. Although no increase in the risk of cancer has been reported so far, these treatments are not recommended for patients with prior history of cancer. ${ }^{37}$ In this study, we systematically explored BMP4 protein expression in a comprehensive collection of normal adult and tumor tissues using immunohistochemistry on tissue microarrays. Moreover, a detailed study of BMP4 expression in a large breast cancer patient set was conducted to evaluate the clinical impact of BMP4 expression in breast cancer. It is noteworthy that the antibody used will detect both the mature BMP4 and the more stable precursor protein, and thus the protein expression data do not directly imply the activity of BMP signaling in a given tissue.

BMP4 protein expression was detected in normal and tumor tissues at variable levels. In more than one-fifth of both the benign and malignant samples, BMP4 was strongly expressed in a granular pattern possibly suggesting a presence in secretory vesicles. 
BMP4 expression was more often absent in normal tissues compared with tumor tissues, while diffuse expression was more frequent in tumors. BMP4 was also commonly expressed in the epithelial cells and leukocytes within both the normal and tumor samples. Although the number of samples studied for each tissue/tumor type was rather low, the BMP4 expression pattern was generally more uniform among normal samples, whereas tumors commonly showed expression patterns representing all four staining categories.

Our extensive array of normal tissues provided a comparable platform to evaluate BMP4 expression and showed that BMP4 is indeed expressed in a distinct set of adult tissues. Thus, BMP4 is likely involved in the normal physiological functions of certain adult tissues. We found strong granular BMP4 staining in the epithelial cells of the bladder, ureter, uterine cervix and the red pulp area of the spleen for the first time. In the lung epithelium, we observed BMP4 expression patterns similar to previous findings. ${ }^{15}$ Strong BMP4 staining was also found in the epithelium of the esophagus and stomach, but a lack of expression was observed in the lower gastrointestinal track (ie, small intestine, colon and rectum). A previous study reported that BMP4 expression was present in inflamed esophageal epithelium and metaplastic Barrett's esophagus but not in normal esophagus. ${ }^{38}$ Likewise, earlier studies reported little or no BMP4 expression in the stomach; however, $H$. pylori infection was shown to increase BMP4 expression in these tissues. ${ }^{28,39}$ Positive BMP4 expression has been previously reported in the normal ovary and endocrine pancreas. ${ }^{12,13}$ On our tissue microarray, these samples were not optimal for the confirmation of these results as no follicles were present in the ovary sections and the endocrine cells were not well represented in the pancreas, which possibly explains the lack of BMP4 expression in these tissues within our sample set. Although direct comparisons between mRNA and protein expression data are not straightforward, public microarray data also demonstrate high BMP4 expression in the urogenital system, gastrointestinal organs and uterine cervix (www.genesapiens.org), thus further corroborating our findings.

The main advantage of our data compared with previous studies is that the tissue microarray format permits the identical handling of all samples, and the scoring of the data from different tissues can be performed according to comparable criteria. Notably, the previous BMP4 expression data from normal adult tissues often stemmed from studies of cancer or other diseases where the normal tissue samples were obtained as paired samples in close proximity of tumor or diseased tissue. This naturally raises some concerns regarding the 'normalness' of the samples. Nevertheless, we fully agree that the number of parallel samples in our study was low; however, their uniform staining patterns still highlights the usefulness of our data.
In cancer samples, strong BMP4 expression was observed in the squamous cell carcinomas. We demonstrate for the first time strong, granular BMP4 expression in the squamous cell carcinomas of the uterine cervix, skin and lung. Our results from head and neck and esophageal squamous cell carcinomas agree with previous findings. ${ }^{30,40,41}$ In addition to these results, the immunohistochemical analysis of BMP4 protein expression in tumor samples has only been performed in colorectal, ovarian, gastric, renal and hepatocellular carcinomas as well as in a few samples of melanoma. In colorectal and gastric cancers, BMP4 expression had been identified in the majority of the samples, but strong expression was detected only in a minority, which resembled the staining pattern we also detected. ${ }^{20,28}$ Our results are also in agreement with previous findings regarding the BMP4 expression in ovarian cancer; ${ }^{29}$ however, in melanoma and renal and hepatocellular carcinomas, strong BMP4 staining has been previously detected more often than our data indicated. ${ }^{19,27,42}$

In this study, we also show that tumor types originating from the same organ or tissue, such as those from lung or skin, can drastically differ in terms of their BMP4 expression. According to earlier reports, BMP4 protein expression was detected in a minority of a small panel of non-small cell lung carcinomas, but studies on the mRNA level of BMP4 did not indicate any differences between the subtypes of lung cancer. ${ }^{43,44}$ In the case of skin, no tumor subtypes besides melanoma had been previously examined. ${ }^{19}$ In the tumor tissue microarray, low levels of BMP4 protein expression were detected in cancers of the prostate and brain that have only been shown to express $B M P 4$ at the mRNA level. ${ }^{45,46}$ For malignancies originating from the thyroid, uterus, spleen and lymph nodes, this is the first study to examine BMP4 protein expression in these tissues.

Similarly to the normal tissues, comparison of our tumor protein expression data and the public mRNA expression data show a good concordance. High $B M P 4$ mRNA expression has been commonly detected for example in lung, gastrointestinal, pancreatic, ovarian and uterine cancers whereas lower expression was seen in prostate carcinomas (www.genesapiens.org). However as stated above, such comparisons are not unproblematic and especially in the case of tumor samples, one may expect more variability in both mRNA and protein levels between different samples.

Finally, the BMP4 expression patterns in the tumor samples were compared with the patterns detected in the corresponding normal tissues. In four organs where BMP4 expression was strong in the normal tissue (ie, stomach, esophagus, liver and spleen), only a subset of the tumor tissue samples expressed BMP4 at a similar level, which implies the expression of BMP4 is reduced in subset of cancers. However, in a previous comparison of 
adjacent normal and tumor tissues from the stomach, liver and esophagus, BMP4 expression was higher in the tumor samples. ${ }^{28,40,47}$ Notably, our normal and tumor tissue microarrays were truly independent samples and not paired unlike previous studies. In the case of four normal tissues (ie, colon, pancreas, thyroid and ovaries) where no BMP4 expression was detected, a subset of the corresponding tumor samples had strong granular BMP4 staining indicating increased BMP4 expression. In colorectal cancer, a similar trend of increased BMP4 expression in tumor tissue has also been observed. ${ }^{20}$ In the prostate and uterine cervix, no differences between the normal and cancer samples were observed, but in the case of brain, lung and skin tissues, the interpretation was not so clear. BMP4 expression in the normal brain tissue was possibly present in the microglial cells, whereas in the tumor tissue, a similar pattern was not evident. Additionally, in the lung, the majority of the tumor samples were similarly granularly stained compared with normal lung with the exception of small cell carcinoma.

For breast cancer, this study is the first report to examine BMP4 protein expression with comprehensive patient material. We have previously shown that $B M P 4$ mRNA is expressed at variable levels in a small panel of primary breast tumors. ${ }^{23,33}$ Similarly to our previous observations, the majority of patient samples also displayed a relatively low BMP4 protein expression level. In $23 \%$ of the samples, BMP4 was expressed in a strong granular manner. The frequency of strong BMP4 expression in breast cancer was similar to the overall frequency observed in other tumor types. In the normal mammary gland tissue, BMP4 was detected only at a low level implying that strong expression is cancer specific. In our previous study, BMP4 mRNA expression was observed more often in low-grade tumors, ${ }^{23}$ but this observation did not translate to the protein level. However, strong BMP4 expression was more common in invasive lobular carcinomas than in ductal carcinomas. We have observed a similar expression pattern for BMP7 in the same breast cancer patient set. ${ }^{35}$ Interestingly, strong BMP4 expression was also observed more often in slowly proliferating tumors, and at the same time, strong BMP4 expression correlated with an increased tumor recurrence. In our previous in-vitro functional studies, BMP4 expression decreased breast cancer cell growth and at the same time was able to increase the migration and invasion of these same cells. $^{23}$ Similarly, BMP4 expression induced an invasive phenotype in a 3D model of normal mammary epithelial cells and reduced their proliferation in concert with other growth factors. ${ }^{48,49}$ Thus, our current data in breast cancer patients confirm these in-vitro observations and suggest that BMP4 is indeed a bidirectional player that harbors both tumorsuppressive and tumor-promoting features in breast cancer. Previously, a low BMP4 methylation score, together with other methylation markers, correlated with a longer time to distant metastasis in breast cancer $^{50}$; however, we did not observe any significant changes in our time-to-event analyses. Overall, the involvement of BMP4 in cancer progression has been studied on a very limited scale. In squamous cell carcinomas of the head and neck, strong BMP4 expression was detected particularly in tumor samples of patients with metastases and was associated with a shorter overall survival. ${ }^{30}$ On the contrary, in ovarian cancer, strong BMP4 expression associated with a longer progression-free time, and in gastric cancer, BMP4 was detected more often in the non-metastatic tumors. ${ }^{28,29}$ Interestingly in the ovarian cancer study, the authors also showed that $B M P 4$ expression is induced by cisplatin treatment but that only the pretreatment level was associated with patient outcome. ${ }^{29}$ These findings emphasize the notion that BMP4's role is most likely highly diverse in different cancer types.

In conclusion, we have shown that BMP4 is expressed in a strong, granular manner in several normal tissues, which indicates that it is likely to have important functions in normal tissue homeostasis. Variable patterns of expression were detected in multiple tumor samples with particularly strong BMP4 staining observed in squamous cell carcinomas. Most importantly, in breast cancer, strong BMP4 expression associated with low proliferation index of the tumors and an increase in tumor recurrence. These observations concur with our previous in-vitro cell line data illustrating a dualistic function for BMP4 as a growth inhibitor and promoter of migration and invasion in breast cancer. A similar dualistic role has been previously established for the superfamily member TGF $\beta$, which functions as a tumor suppressor during the first steps of tumor formation, but it is later found to stimulate cancer metastasis. ${ }^{51,52}$

\section{Acknowledgements}

This study was partly supported by grants from the Sigrid Juselius Foundation, the Competitive Research Funding of the Tampere University Hospital, and the Finnish Cancer Organizations. Professor Timo Paavonen is acknowledged for his contribution in the BMP4 immunohistochemistry. The skillful assistance of laboratory technician Eini Eskola and research nurse Kirsi Rouhento is greatly appreciated.

\section{Disclosure/conflict of interest}

The authors declare no conflict of interest.

\section{References}

1 Miyazono K, Kamiya Y, Morikawa M. Bone morphogenetic protein receptors and signal transduction. J Biochem 2012;147:35-51. 
2 Reddi AH. Bone morphogenetic proteins: an unconventional approach to isolation of first mammalian morphogens. Cytokine Growth Factor Rev 1997;8: 11-20.

3 Zhao GQ. Consequences of knocking out BMP signaling in the mouse. Genesis 2003;35:43-56.

4 Winnier G, Blessing M, Labosky PA, et al. Bone morphogenetic protein-4 is required for mesoderm formation and patterning in the mouse. Genes Dev 1995;9:2105-2116.

5 Danesh SM, Villasenor A, Chong D, et al. BMP and BMP receptor expression during murine organogenesis. Gene Expr Patterns 2009;9:255-265.

6 Hogan BL. Bone morphogenetic proteins: multifunctional regulators of vertebrate development. Genes Dev 1996;10:1580-1594.

7 Robinson GW. Cooperation of signalling pathways in embryonic mammary gland development. Nat Rev Genet 2007;8:963-972.

8 Schmierer B, Hill CS. TGFbeta-SMAD signal transduction: molecular specificity and functional flexibility. Nat Rev Mol Cell Biol 2007;8:970-982.

9 Sieber C, Kopf J, Hiepen C, et al. Recent advances in BMP receptor signaling. Cytokine Growth Factor Rev 2009;20:343-355.

10 Bragdon B, Moseychuk O, Saldanha S, et al. Bone morphogenetic proteins: a critical review. Cell Signal 2011;23:609-620.

11 Nakase T, Yoshikawa H. Potential roles of bone morphogenetic proteins (BMPs) in skeletal repair and regeneration. J Bone Miner Metab 2006;24:425-433.

12 Otsuka F. Multiple endocrine regulation by bone morphogenetic protein system. Endocr J 2010;57:3-14.

13 Goulley J, Dahl U, Baeza N, et al. BMP4-BMPR1A signaling in beta cells is required for and augments glucose-stimulated insulin secretion. Cell Metab 2007;5:207-219.

14 Paulson RF, Shi L, Wu DC. Stress erythropoiesis: new signals and new stress progenitor cells. Curr Opin Hematol 2011;18:139-145.

15 Myllarniemi M, Vuorinen $\mathrm{K}$, Pulkkinen $\mathrm{V}$, et al. Gremlin localization and expression levels partially differentiate idiopathic interstitial pneumonia severity and subtype. J Pathol 2008;214:456-463.

16 Badreddine RJ, Wang KK. Barrett esophagus: an update. Nat Rev Gastroenterol Hepatol 2010;7:369-378.

17 Singh A, Morris RJ. The yin and yang of bone morphogenetic proteins in cancer. Cytokine Growth Factor Rev 2010;21:299-313.

18 Buckley S, Shi W, Driscoll B, et al. BMP4 signaling induces senescence and modulates the oncogenic phenotype of A549 lung adenocarcinoma cells. Am J Physiol Lung Cell Mol Physiol 2004;286:L81-L86.

19 Rothhammer T, Poser I, Soncin F, et al. Bone morphogenic proteins are overexpressed in malignant melanoma and promote cell invasion and migration. Cancer Res 2005;65:448-456.

20 Deng H, Makizumi R, Ravikumar TS, et al. Bone morphogenetic protein-4 is overexpressed in colonic adenocarcinomas and promotes migration and invasion of HCT116 cells. Exp Cell Res 2007;313:1033-1044.

21 Hamada S, Satoh K, Hirota M, et al. Bone morphogenetic protein 4 induces epithelial-mesenchymal transition through MSX2 induction on pancreatic cancer cell line. J Cell Physiol 2007;213:768-774.

22 Theriault BL, Shepherd TG, Mujoomdar ML, et al. BMP4 induces EMT and rho GTPase activation in human ovarian cancer cells. Carcinogenesis 2007;28: 1153-1162.

23 Ketolainen JM, Alarmo EL, Tuominen VJ, et al. Parallel inhibition of cell growth and induction of cell migration and invasion in breast cancer cells by bone morphogenetic protein 4. Breast Cancer Res Treat 2010;124:377-386.

24 Wahdan-Alaswad RS, Song K, Krebs TL, et al. Insulinlike growth factor I suppresses bone morphogenetic protein signaling in prostate cancer cells by activating mTOR signaling. Cancer Res 2010;70:9106-9117.

25 Virtanen S, Alarmo EL, Sandstrom S, et al. Bone morphogenetic protein -4 and -5 in pancreatic cancernovel bidirectional players. Exp Cell Res 2011;317: 2136-2146.

26 Houlston RS, Webb E, Broderick P, et al. Meta-analysis of genome-wide association data identifies four new susceptibility loci for colorectal cancer. Nat Genet 2008;40:1426-1435.

27 Kwak C, Park YH, Kim IY, et al. Expression of bone morphogenetic proteins, the subfamily of the transforming growth factor-beta superfamily, in renal cell carcinoma. J Urol 2007;178:1062-1067.

28 Kim SG, Park HR, Min SK, et al. Expression of bone morphogenic protein-4 is inversely related to prevalence of lymph node metastasis in gastric adenocarcinoma. Surg Today 2011;41:688-692.

29 Laatio L, Myllynen P, Serpi R, et al. BMP-4 expression has prognostic significance in advanced serous ovarian carcinoma and is affected by cisplatin in OVCAR-3 cells. Tumour Biol 2011;32:985-995.

$30 \mathrm{Xu} \mathrm{T}, \mathrm{Yu} \mathrm{CY}$, Sun JJ, et al. Bone morphogenetic protein4-induced epithelial-mesenchymal transition and invasiveness through Smad1-mediated signal pathway in squamous cell carcinoma of the head and neck. Arch Med Res 2011;42:128-137.

31 Chiu CY, Kuo KK, Kuo TL, et al. The activation of MEK/ERK signaling pathway by bone morphogenetic protein 4 to increase hepatocellular carcinoma cell proliferation and migration. Mol Cancer Res 2012;10: 415-427.

32 Alarmo EL, Kallioniemi A. Bone morphogenetic proteins in breast cancer: Dual role in tumourigenesis? Endocr Relat Cancer 2010;17:R123-R139.

33 Alarmo EL, Kuukasjarvi T, Karhu R, et al. A comprehensive expression survey of bone morphogenetic proteins in breast cancer highlights the importance of BMP4 and BMP7. Breast Cancer Res Treat 2007;103: 239-246.

34 Korhonen T, Huhtala H, Holli K. A comparison of the biological and clinical features of invasive lobular and ductal carcinomas of the breast. Breast Cancer Res Treat 2004;85:23-29.

35 Alarmo EL, Korhonen T, Kuukasjarvi T, et al. Bone morphogenetic protein 7 expression associates with bone metastasis in breast carcinomas. Ann Oncol 2008;19:308-314.

36 Kelleher FC, Fennelly D, Rafferty M. Common critical pathways in embryogenesis and cancer. Acta Oncol 2006;45:375-388.

37 Thawani JP, Wang AC, Than KD, et al. Bone morphogenetic proteins and cancer: review of the literature. Neurosurgery 2010;66:233-246.

38 Milano F, van Baal JW, Buttar NS, et al. Bone morphogenetic protein 4 expressed in esophagitis induces a columnar phenotype in esophageal squamous cells. Gastroenterology 2007;132:2412-2421. 
39 Bleuming SA, Kodach LL, Garcia Leon MJ, et al. Altered bone morphogenetic protein signalling in the helicobacter pylori-infected stomach. J Pathol 2006;209:190-197.

40 van Baal JW, Milana F, Rygiel AM, et al. A comparative analysis by SAGE of gene expression profiles of esophageal adenocarcinoma and esophageal squamous cell carcinoma. Cell Oncol 2008;30:63-75.

41 Strnad H, Lacina L, Kolar M, et al. Head and neck squamous cancer stromal fibroblasts produce growth factors influencing phenotype of normal human keratinocytes. Histochem Cell Biol 2010;133:201-211.

42 Guo X, Xiong L, Zou L, et al. Upregulation of bone morphogenetic protein 4 is associated with poor prognosis in patients with hepatocellular carcinoma. Pathol Oncol Res 2012;18:635-640.

43 Langenfeld EM, Bojnowski J, Perone J, et al. Expression of bone morphogenetic proteins in human lung carcinomas. Ann Thorac Surg 2005;80:1028-1032.

44 Bieniasz M, Oszajca K, Eusebio M, et al. The positive correlation between gene expression of the two angiogenic factors: VEGF and BMP-2 in lung cancer patients. Lung Cancer 2009;66:319-326.

45 Bentley H, Hamdy FC, Hart KA, et al. Expression of bone morphogenetic proteins in human prostatic adenocarcinoma and benign prostatic hyperplasia. Br J Cancer 1992;66:1159-1163.
46 Piccirillo SG, Reynolds BA, Zanetti N, et al. Bone morphogenetic proteins inhibit the tumorigenic potential of human brain tumour-initiating cells. Nature 2006;444:761-765.

47 Maegdefrau U, Amann T, Winklmeier A, et al. Bone morphogenetic protein 4 is induced in hepatocellular carcinoma by hypoxia and promotes tumour progression. J Pathol 2009;218:520-529.

48 Montesano R. Bone morphogenetic protein-4 abrogates lumen formation by mammary epithelial cells and promotes invasive growth. Biochem Biophys Res Commun 2007;353:817-822.

49 Montesano R, Sarkozi R, Schramek H. Bone morphogenetic protein-4 strongly potentiates growth factorinduced proliferation of mammary epithelial cells. Biochem Biophys Res Commun 2008;374:164-168.

50 Hartmann O, Spyratos F, Harbeck N, et al. DNA methylation markers predict outcome in nodepositive, estrogen receptor-positive breast cancer with adjuvant anthracycline-based chemotherapy. Clin Cancer Res 2009;15:315-323.

51 Siegel PM, Massague J. Cytostatic and apoptotic actions of TGF-beta in homeostasis and cancer. Nat Rev Cancer 2003;3:807-821.

52 Massague J, Gomis RR. The logic of TGFbeta signaling. FEBS Lett 2006;580:2811-2820.

Supplementary Information accompanies the paper on Modern Pathology website (http://www.nature.com/ modpathol) 